\title{
Corneal hydrops induced by diabetic ketoacidosis: A case report
}

\author{
MEIYAN FENG and CHAO WANG \\ Department of Ophthalmology, Zaozhuang Municipal Hospital, Zaozhuang, Shandong 277000, P.R. China
}

Received May 28, 2015; Accepted January 15, 2016

DOI: $10.3892 /$ etm.2016.3524

\begin{abstract}
To the best of our knowledge, there have been no reports of corneal hydrops associated with diabetic ketoacidosis. The present study first reports a case of a 20 -year-old male patient with diabetic ketoacidosis-induced corneal hydrops. The patient exhibited mild hydrops in their left eyelid, which was accompanied by mixed hyperemia, and hazy turbid in a white color was observed in the cornea. To alleviate the corneal hydrops, $5 \%$ glucose was administered dropwise to the left eye for $2 \mathrm{~h}$ to alleviate the inflammation. Finally, the patient was discharged from the hospital with a satisfactory outcome.
\end{abstract}

\section{Introduction}

Diabetic ketoacidosis, a life-threatening complication of diabetes mellitus (DM), occurs with the accumulation of glucose due to insulin insufficiency (1). As one of the acute complications of diabetes mellitus, diabetic ketoacidosis causes great threats to patients. Corneal hydrops are characterized by stromal edema, which is the result of leakage of aqueous through a tear in Descemet's membrane (2). Studies have identified risk factors for developing acute hydrops, such as steeper keratometry and poorer Snellen visual acuity at the time of diagnosis $(2,3)$. However, to the best of our knowledge, no cases of diabetic ketoacidosis-induced corneal hydrops have been reported. This case report described the case of corneal hydrops resulting from diabetic ketoacidosis in a 20 -year-old male patient.

\section{Case report}

A 20-year-old male patient was admitted to the Department of Endocrinology of the Zaozhuang Municipal Hospital (Zaozhuang, China) on July 31, 2013 presenting with diabetic ketoacidosis. The patient reported inflammation in the left

Correspondence to: Professor Meiyan Feng, Department of Ophtalmology, Zaozhuang Municipal Hospital, 41 Longtou Road, Zaozhuang, Shandong 277000, P.R. China

E-mail: fengmy66@163.com

Key words: corneal hydrops, diabetic ketoacidosis, corneal endothelium, diabetes mellitus eye, a foreign body sensation in both eyes and impaired visual acuity for 3 days, together with palpitation and shortness of breath for $3 \mathrm{~h}$. The present study was approved by the Ethics Committee of Zaozhuang Municipal Hospital and informed consent was obtained from the patient.

The physical examination revealed no abnormality. The patient was conscious but in a depressed mood. In addition, an odor described as resembling 'rotten apples' was detected during deep respiration. Poor skin elasticity was manifested. Rough sounds were heard in the lungs upon breathing, with no dry or wet rales and a heart rate of $126 \mathrm{bpm}$. Low skin temperature was detected in the distal end of the limbs and no hydrops was observed in either of the lower extremities. Weak arterial pulse was noted on the dorsa of the feet. No Babinski sign was identified. Ophthalmology consultation was performed due inhibited vision in the left eye. The ophthalmological examination indicated that light perception was present in the visual acuity of the left eye, and intraocular pressure was found to be $13.0 \mathrm{mmHg}$. Mild swelling was observed in the eyelid, along with mixed hyperemia. Gray, homogeneous haze was observed in the cornea, which led to the diagnosis of diabetic ketoacidosis-induced corneal hydrops (Fig. 1).

Following admission to the hospital, dual-channel fluid infusion (administration of $0.9 \%$ sodium chloride followed by $5 \%$ glucose) was performed to replenish blood volume. A small dose of insulin (22 units; Novo Nordisk Co., Ltd., Beijing, China) was persistently used to inhibit steatolysis and ketoplasia. Acid-base disorders were corrected and water-electrolyte balance was maintained. In addition, blood sugar monitoring was enhanced, and electrolyte and amylase tests, blood lactic acid and creatase detection, as well as electrocardiogram were performed to determine the patient's condition. In order to alleviate the corneal hydrops, $50 \%$ glucose was administered dropwise into the left eye for $30 \mathrm{~min}$, followed by the administration of TobraDex $(30 \mu 1$; Alcon, Inc., Fort Worth, TX, USA) for $2 \mathrm{~h}$ to alleviate the inflammation. Approximately $4 \mathrm{~h}$ after treatment, the visual acuity and intraocular pressure of the left eye were 0.08 and $12 \mathrm{mmHg}$, respectively. Mixed hyperemia abated and mild hydrops was noted in the cornea. A deep anterior chamber with turbid aqueous humor was observed (++). In addition, $7 \mathrm{~h}$ later adhesions were noted in the pupil. The diameter of the pupil measured $\sim 2 \mathrm{~mm}$, the lenses were transparent and fundus oculi relatively normal. Atropine sulfate eye gel (tid; $25 \mathrm{mg}$; Xingqi Pharmacy, Shenyang, China), pranoprofen (5 mg; Senju Pharmaceutical Co., Ltd., Tokyo, Japan) ophthalmic solution (q2h; Alcon, Inc.) and tobramycin $(80 \mu \mathrm{l})$ and dexamethasone 


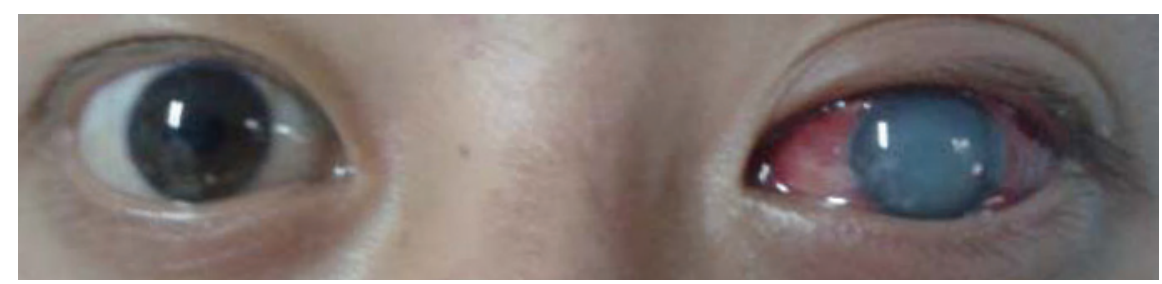

Figure 1. A 20 -year-old male patient was admitted to Zaozhuang Municipal Hospital hospital presenting with corneal hydrops. The patient had been suffering from diabetic ketoacidosis 9 years. A disorder of glucose metabolism, caused by insulin insufficiency, was observed.

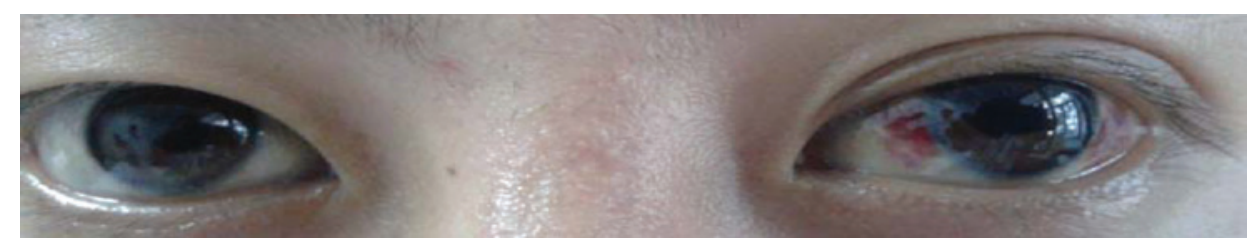

Figure 2. Attenuation of corneal hydrops was observed following perfusion of the insulin and improvement the microcirculation. The dosage of insulin was modulated in order to control the blood sugar, and potassium was injected to connect the higher blood glucose and the acidosis.

$(1 \mathrm{ml})$ (both purchased from Qilu Pharmacy) eye ointment (qn) were administered. On the next day following admission, the visual acuity of the left eye was $0.8 \mathrm{mmHg}$ and the intraocular pressure was maintained at $14 \mathrm{mmHg}$. Slightly mixed hyperemia was observed, and the transparency of the cornea appeared to have been restored (Fig. 2). The anterior eye ball chamber was deep, with mild turbid aqueous humor. The patient was discharged from the Department of Ophtalmology with a satisfactory treatment outcome.

\section{Discussion}

The typical symptoms of corneal endothelium are characterized by hydrops and opacity in the corneal endothelial cells and cornea (4). No direct association has been established between corneal endothelium and intraocular inflammation-induced corneal endothelial syndromes. In addition, the immunological reactive proteins released in the presence of iridocyclitis could enter the anterior chamber, leading to the apoptosis of corneal endothelial cells and the descent of cellular functions (5). According to a previous study, the uveitis associated with DM was primarily manifested as anterior uveitis with a unilateral onset (6). Furthermore, the incidence of retinopathy was rarely identified in these cases (6). In the present study, normal visual acuity was obtained once the iridocyclitis was controlled in the patient, therefore confirming that the iridocyclitis was induced by DM.

Physiologically, corneal normal thickness and transparency are maintained by the barrier function of corneal endothelial cells and active transport (7). Among the perfusate of endothelial cells, sodium and bicarbonate are necessary. Bicarbonate deficiency resulted in corneal hydrops (7). In addition, the carbonic anhydrase inhibitor in endothelial cells is able to attenuate the speed of the bicarbonate release from the aqueous humor, which finally induced mild corneal hydrops (8). Prior to treatment in the present study, the bicarbonate level was $3.4 \mathrm{mmol} / \mathrm{l}$, markedly lower than the normal level (22-29 mmol/l); however, following the correction of the acidosis, normal corneal transparency was restored and the visual acuity reached $0.8 \mathrm{mmHg}$. On this basis, we speculated that the corneal hydrops had been caused by a low bicarbonate level that affected the pumping functions of the corneal endothelial.

In order to maintain the barrier and active transport functions of corneal endothelial cells, the cells covered the posterior surface of the cornea completely (9). Endothelial cell pleomorphism change was defined as a type of injury mechanism that normal endothelial cell locomotion repaired injured regions (10). The pleomorphism ratio of endothelial cells was expressed by the coefficient of variation (CV) of the cellular area (normal value, $0.22-0.31$ ). When the $\mathrm{CV}$ exceeded 0.40 , abnormalities were observed. The $\mathrm{CV}$ of this patient was $33.2 \%$.

It was found that among perfusate for endothelial cells, sodium and bicarbonate were necessary. For example, bicarbonate deficiency led to corneal hydrops. When the patient was admitted to the hospital, his $\mathrm{HCO}_{3}{ }^{2-}$ level $(19 \mathrm{mmol} / \mathrm{l})$ was lower than normal, affecting corneal pumping functions that resulted in corneal hydrops. Following admission, dual-channel fluid infusion was performed, using a total volume of $2,800 \mathrm{ml}$, which consisted of salt water $(1,700 \mathrm{ml})$, $5 \%$ glucose $(1,000 \mathrm{ml})$ and $5 \% \mathrm{SB}(100 \mathrm{ml})$. Potassium chloride was administered at a dose of $4.5 \mathrm{~g}$, along with an intravenous pancreatic islet (22 $\mathrm{U})$ and water $(\sim 1,500 \mathrm{ml})$. On the day after admission, acidosis was corrected and visual acuity returned to $0.8 \mathrm{mmHg}$.

To the best of our knowledge, rare cases have been reported to suffer from corneal hydrops due to ketoacidosis. In the present case report, the patient was diagnosed with multiple diabetic ketoacidosis. In addition, anterior uveitis was identified in the left eye. No treatment had been administered to the patient prior to his admission to the Zaozhuang Municipal Hospital, due to lack of severe lesions. The patient had been suffering from DM for nearly 9 years. Previously, the patient had been admitted to hospitals, presenting with diabetic ketoacidosis. Prior to admission to our hospital, the patient exhibited severe corneal hydrops together with abnormalities in the eyelid and bulbar conjunctiva hyperemia. Furthermore, severe 
insulin insufficiency was suspected together with metabolic disorder of blood sugar in vivo. Simultaneously, a large quantity of fat was degenerated, leading to an excessive accumulation of acetone bodies. In combination, all these symptoms may eventually induce diabetic ketoacidosis. During hospitalization, symptomatic treatment to attenuate the disease condition was performed in order to perfuse the insulin and improve the microcirculation. Subsequently, the concentration of acetone in the body decreased. The dosage of insulin pump was modulated in order to control the blood sugar. Potassium was then injected to correct the higher blood glucose and acidosis. The patient was finally discharged with a satisfactory outcome.

In conclusion, the present study reported the first case of corneal hydrops induced by diabetic ketoacidosis. Following symptomatic treatment, the patient was discharged with a satisfactory outcome. This case report could provide helpful insight in the clinical management of patients with similar conditions.

\section{References}

1. Westerberg DP: Diabetic ketoacidosis: evaluation and treatment. Am Fam Physician 87: 337-346, 2013.
2. Tuft SJ, Gregory WM and Buckley RJ: Acute corneal hydrops in keratoconus. Ophthalmology 101: 1738-1744, 1994.

3. Fan Gaskin JC, Patel DV and McGhee CN: Acute corneal hydrops in keratoconus - new perspectives. Am J Ophthalmol 157: 921-928, 2014

4. Wollensak G, Sporl E, Reber F, Pillunat L and Funk R: Corneal endothelial cytotoxicity of riboflavin/UVA treatment in vitro. Ophthalmic Res 35: 324-328, 2003.

5. Underwood PA, Bennett FA, Mott MR and Strike P: Collagen-associated molecules in the cornea: Localisation with monoclonal antibodies. Exp Eye Res 58: 139-153, 1994.

6. Squirrell DM, Bhatta S, Mudhar HS and Rennie IG: Hypertensive iridocyclitis associated with delayed onset biopsy proven Cytomegalovirus retinitis. Indian J Ophthalmol 65 656-658, 2013

7. Edelhauser HF: The balance between corneal transparency and edema: The Proctor Lecture. Invest Ophthalmol Vis Sci 47: 1754-1767, 2006

8. Wu NC, Chiang $\mathrm{CH}$ and Lee AR: Studies of carbonic anhydrase inhibitors: Physicochemical properties and bioactivities of new thiadiazole derivatives. J Ocul Pharmacol 9: 97-108, 1993.

9. Franzen AA, Pigatto JA, Abib FC, Albuquerque L and Laus JL: Use of specular microscopy to determine corneal endothelial cell morphology and morphometry in enucleated cat eyes. Vet Ophthalmol 13: 222-226, 2010.

10. Esgin H and Erda N: Corneal endothelial polymegethism and pleomorphism induced by daily-wear rigid gas-permeable contact lenses. CLAO J 28: 40-43, 2002. 